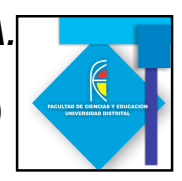

\title{
RECONTEXTUALIZACIÓN EN LA ENSEÑANZA DEL CONCEPTO DE GRAVEDAD A PARTIR DE UN ANÁLISIS HISTORICO-EPISTEMOLÓGICO DE LA PERSPECTIVA GALILEANA
}

\section{RECONTEXTUALIZATION IN TEACHING THE CONCEPT OF GRAVITY FROM A HISTORICAL EPISTEMOLOGICAL ANALYSIS OF THE GALILEAN PERSPECTIVE}

\section{Resumen}

Isabel Cristina Machado ${ }^{1}$ Eliana Andrea Restrepo ${ }^{2}$ Isned Elena Sossa ${ }^{3}$

Yirsen Aguilar Mosquera ${ }^{4}$

En los análisis realizados en algunas investigaciones y en el contexto de la práctica profesional, se ha logrado evidenciar que, usualmente la enseñanza de la física se centra en la solución de algoritmos planteados como ejercicios que, en ningún caso, se ocupan de la conceptualización y contextualización de los conceptos estructurantes de la Mecánica, como caso particular se puede referenciar la Segunda ley de Newton y su relación con la caída de los cuerpos.

En el caso de la caída libre se parte de la expresión $\boldsymbol{F}=\boldsymbol{m a}$ y, se concluye que $\boldsymbol{F}=\boldsymbol{m} \boldsymbol{g}$. Al despejar dicha expresión, la gravedad se asume como una variable que depende de la masa. Esta situación, lleva a que el concepto de gravedad y fuerza gravitacional se asuman indistintamente, es decir, se confunda la fuerza gravitacional y la aceleración de la gravedad.

Se plantea entonces que a estas maneras de significar y de enseñar estos conceptos, subyace un modo particular de asumir la Ciencia y la Enseñanza, y en tal sentido, se adelanta un análisis histórico y epistemológico de la perspectiva galileana, por considerar que su particular manera de abordar y formalizar el fenómeno de la caída de los cuerpos en su obra Consideraciones y Demostraciones Matemáticas sobre Dos Nuevas Ciencias (1976) , posibilita, no solo explicitar maneras de hacer ciencia sino que, además, permite recontextualizar y re significar el concepto de aceleración de gravedad y fuerza

\footnotetext{
${ }^{1}$ Estudios Culturales sobre las Ciencias y su Enseñanza - ECCE-Facultad de Educación, Universidad de Antioquia. Medellín, Colombia.Icri_19@yahoo.com

${ }^{2}$ Estudios Culturales sobre las Ciencias y su Enseñanza - ECCE-Facultad de Educación, Universidad de Antioquia. Medellín, , elyandrea883@gmail.com

${ }^{3}$ Estudios Culturales sobre las Ciencias y su Enseñanza - ECCE-Facultad de Educación, Universidad de Antioquia. Medellín, , isnedsossa@yahoo.es

${ }^{4}$ Estudios Culturales sobre las Ciencias y su Enseñanza - ECCE-Facultad de Educación, Universidad de Antioquia. Medellín, C yirsena@yahoo.es
} 


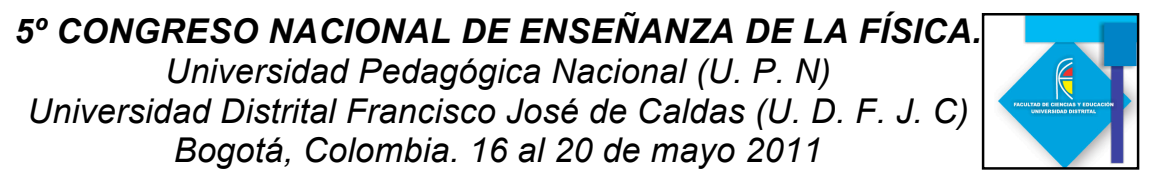

gravitacional. En este análisis se muestra cómo Galileo establece una

diferencia entre gravedad y fuerza gravitacional, considera a la gravedad como un efecto (aceleración) y a la fuerza gravitacional como la acción que genera los cambios de velocidad en un cuerpo. Se analiza además cómo el uso de la Geometría en la organización del fenómeno del movimiento, permite a Galileo dar forma a esta clase de fenómenos, aspecto que se constituye en una fructífera manera de significar la relación entre la física y las matemáticas.

Finalmente, se plantean unas implicaciones didácticas que surgen como producto del análisis de la perspectiva galileana y de los datos obtenidos con la aplicación de algunos instrumentos a cinco casos de la Institución Educativa Comercial de Envigado.

Palabras clave: Gravedad, caída libre, conceptualización, recontextualización, historia y epistemología, fenomenología, formalización.

\section{Abstract}

In the analysis performed in some research and in the context of professional practice, it has been evident, usually physics teaching focuses on the solution algorithms proposed as exercises in any case, dealing with the conceptualization andcontextualization of the structural concepts of mechanics as a particular case can be referenced Newton's second law and its relationship with the falling bodies.

In the case of free fall is part of the expression $\mathrm{F}=\mathrm{ma}$ and concluded that $\mathrm{F}=\mathrm{mg}$.Solving this expression, the gravity is assumed as a variable that depends on the mass. This situation leads to the concept of gravity and gravitational embrace either, that is, they confuse the gravitational force and the acceleration of gravity.

Then arises that these forms of meaning and to teach these concepts, underlying a particular way to take the Science and Education, and in that sense, come on historical and epistemological analysis of the Galilean perspective, arguing that their particular way to address and formalize the phenomenon of falling bodies in his considerations and Mathematics Demonstrations on Two New Sciences (1976), allows not only ways to explain science but also allows reframing and re mean the concept of acceleration of gravity and gravitational force. This analysis shows how Galileo distinguishes between gravity and gravitational force, considered as an effect of gravity (acceleration) and the gravitational force as the action that generates speed changes in a body. It also examines how the use of geometry in the organization of the phenomenon of movement, allowing Galileo to shape this kind of phenomena, an aspect which constitutes a fruitful way to signify the relationship between physics and mathematics.

Finally, it presents some pedagogical implications which arise as a result of the analysis of the Galilean perspective and data from the application of some instruments to five cases of School Business Envigado. 
Keywords: gravity, free fall, conceptualization, recontextualization, history and epistemology, phenomenology, formalization.

\section{El concepto de gravedad en el contexto de la enseñanza}

En análisis realizados a investigaciones en torno a la enseñanza de la física, se ha puesto de manifiesto que habitualmente el análisis de los fenómenos físicos se realiza a través de las matemáticas, como la estructura formal de la física, utilizando y adaptando esta estructura para la comprensión y organización de las explicaciones físicas. Es de esta forma que surgen los algoritmos matemáticos como una forma de explicar determinados fenómenos físicos, en los cuales se ponen en juego variables que se relacionan para resolver situaciones físicas, en las que se conocen algunos datos.

No obstante, y acorde con estos análisis en algunos libros de texto y en la práctica profesional, igualmente, se ha podido evidenciar que dichos algoritmos se postulan como ejercicios que su solución planea resolver algoritmos que en pocos casos involucra una reflexión sobre la conceptualización y contextualización de los fenómenos físicos, es el caso de la caída libre de los cuerpos, la cual se aborda desde la relación que se establece con la segunda ley de Newton. En algunos textos escolares, se plantea la ecuación de caída libre como consecuencia de la segunda ley de Newton, sin especificar cuáles son las condiciones necesarias para que se pueda dar esta comparación, Valero afirma que "Cuando un cuerpo cae libremente, su aceleración es la de la gravedad $\mathrm{a}=\mathrm{g}$ y la fuerza que actúa sobre él es su peso $\mathrm{F}=\mathrm{w}$, por tanto, la segunda ley de Newton $\mathrm{F}=$ ma, queda como $\mathrm{W}=\mathrm{mg}^{\prime \prime}(2002, \mathrm{p}$ 102). Puede pensarse que si previamente no se adelanta una reflexión sobre lo que implica esta expresión, fácilmente los estudiantes al despejar la gravedad de esta ecuación, llegan a que: $\mathrm{g}=\mathrm{w} / \mathrm{m}$, de lo que infieren que la aceleración de caída (gravedad) depende de la masa del cuerpo.

¿Cómo entender entonces que la gravedad es constante y que es independiente de la masa?

Como consecuencia de lo anterior se genera dificultades de orden conceptual como: "los cuerpos, cuanto más pesados son, antes llegan al suelo, idea que lleva a pensar que si un cuerpo pesa el doble que otro, cuando se dejen caer desde la misma altura, el primero empleará en llegar al suelo justo la mitad de tiempo que el segundo, o que cuando se lancen verticalmente hacia arriba con la misma velocidad, el primero alcanzará justamente la mitad de altura que el segundo". (Carrascosa, 2006, pp. 77-88) ${ }^{5}$. Como consecuencia de lo anterior se infiere que los cuerpos de mayor masa son más atraídos que los de menor masa.

Puede afirmarse entonces que no es suficiente solamente la enseñanza de las formulas matemáticas sino también la adecuada conceptualización y las respectivas idealizaciones

${ }^{5}$ Carrascosa J. El problema de las concepciones alternativas en la actualidad (parte III). Utilización didáctica de los errores conceptuales que aparecen en cómics, prensa, novelas y libros de texto. Rev. Eureka. Enseñanza y Divulgación de las Ciencias., 2006, 3(1), pp. 77-88 


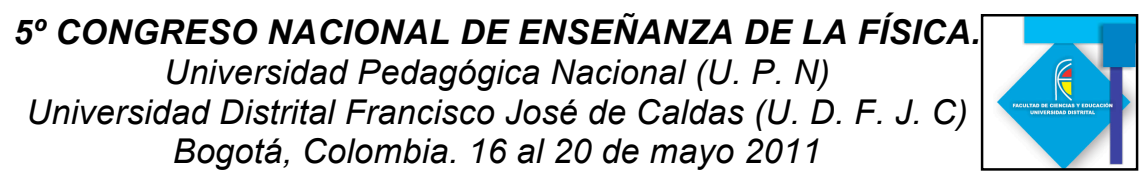

que permiten la construcción del conocimiento científico. En consecuencia, analizar un fenómeno implica identificar cuáles son las condiciones que hacen posible su ocurrencia,

es decir, bajo que circunstancia se ha hecho posible su consolidación como conocimiento en la Ciencia.

Consecuente con esto se adelanta un análisis de la manera como Galileo formaliza la caída de los cuerpos, por considerar que su particular manera de formalizar este fenómeno no sólo posibilita su comprensión sino que además permite plantear alternativas didácticas que muestran una relación entre la matemática y la física, en donde tanto el componente matemático como la organización de fenomenologías emergen simultáneamente.

\section{La caída de los cuerpos en la perspectiva galileana.}

\section{Los experimentos mentales en la construcción de fenomenologías.}

Galileo en la construcción de sus postulados, se vale de ciertos procesos mentales, en los que realiza una serie de experimentos bajo condiciones ideales, como planos inclinados perfectamente lisos o la no existencia de resistencia de algún medio. Estos procesos mentales surgen de conocimientos ya estructurados en el pensamiento, es decir de una experimentación mental, a partir de la cual crea unas condiciones fijas y verdaderas que se cumplen en su teoría física a las que denomina definiciones, bajo las cuales se da la ocurrencia de los fenómenos, posibilitando de esta manera la creación de una estructura metodológica, que se establece en la utilización de unas definiciones, axiomas, teoremas y proposiciones para dar organización a los fenómenos.

Es así como las definiciones nacen de la experiencia y son el sustento teórico que garantiza la ocurrencia de los fenómenos, estas definiciones dan pie a unos axiomas que son también condiciones iníciales las cuales dan forma a unas proposiciones que le permiten ejemplificar o expresar geométricamente la ciencia del movimiento.

En la perspectiva galileana, en el movimiento de caída los grados de velocidad van aumentando a medida que transcurre el tiempo, de modo que el aumento de los grados de velocidad en el tiempo son constantes, planteando de esta manera un movimiento uniformemente acelerado, donde la masa no influye en la velocidad de caída de los cuerpos, más bien establece que es la diferencia de densidades entre el medio y el cuerpo lo que determina el grado de velocidad de caída del cuerpo, además considera que puede existir un medio en que la densidad sea cero, es decir un espacio vacío, donde la caída no va depender de la masa del cuerpo.

\section{El papel de la experiencia sensible en la organización de fenomenologías.}

En la formalización del movimiento, Galileo no solo utiliza la experimentación mental como estrategia metodológica, sino que además en sus postulados, juega un papel importante la experiencia sensible, debido a que sus constructos teóricos están permeados por la 


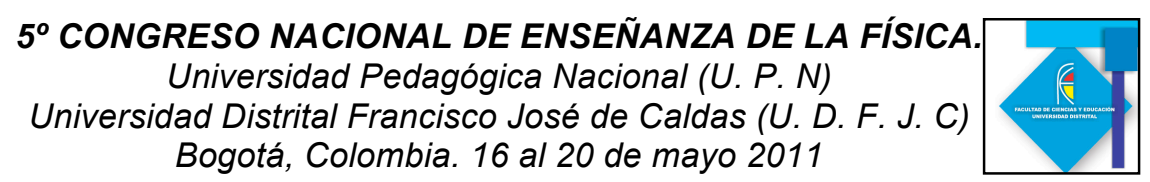

forma en la que interactú con el mundo exterior; su forma de representarlo habla de su propia visión del mundo físico.

Esto se ejemplifica cuando plantea que para poder hablar de movimiento uniformemente acelerado, se debe observar primero la caída de los cuerpos que ocurre de manera

natural, tomándose la caída de los cuerpos como prototipo para la conceptualización de dicho movimiento: (Galilei, 1976, p 275)

... desde el momento que la naturaleza se sirve de una determinada forma de aceleración, en los cuerpos pesados en caída libre, hemos decidido estudiar sus propiedades haciendo que la definición que hemos de dar acerca del movimiento acelerado en cuestión corresponda a la esencia del movimiento naturalmente acelerado.

De aquí es posible inferir en Galileo la importancia de la experiencia sensible en los postulados que dan origen a su teoría del movimiento y en especial al concepto de aceleración en el fenómeno de caída libre de los cuerpos.

\section{Relación física-matemática en la formalización del fenómeno de caída libre.}

Galileo en sus fundamentos establece unas relaciones proporcionales en las variables físicas que intervienen en los fenómenos, considerando los objetos como construcciones matemáticas, donde estas relaciones generan proposiciones matemáticas que a su vez explican un fenómeno físico, siendo estas relaciones constitutivas, ya que no se concibe las construcciones físicas separadas de las construcciones matemáticas.

Dentro de la matematización de los fenómenos físicos, Galileo representa por medio de longitudes las variables que él considera, permiten explicar el fenómeno de caída libre (tiempo, velocidad y gravedad). Esto lo hace también con el fin de relacionar variables de la misma clase al ponerlas todas en el lenguaje de las longitudes. De esta manera, se videncia una relación de constitución entre la física y la matemática, en el sentido de que las dos coexisten de manera tributaria en la formalización de los fenómenos.

\section{Implicaciones Didácticas.}

Al ser abordados los fenómenos desde la perspectiva galileana, en particular el de la caída libre de los cuerpos, es posible brindar a los estudiantes una manera de conceptualizar donde se da forma a los fenómenos físicos y a su vez se construye una estructura matemática desde relaciones de proporcionalidad, facilitando el aprendizaje de la física, ya que una de las mayores dificultades en el aprendizaje se encuentra el manejo que se le da a las matemáticas y si esta se construye, junto con la comprensión de una fenómeno estudiado, facilita la apropiación del conocimiento por parte de los estudiantes.

Al utilizar los diálogos, Galileo inicia desde el pensamiento aristotélico de la época llevando progresivamente al lector a identificar las posibles falencias que tiene dicha visión, abriendo paso a sus explicaciones del mundo físico, que se cumplen bajo ciertas Revista Científica . Volumen Extral. Año 2011 


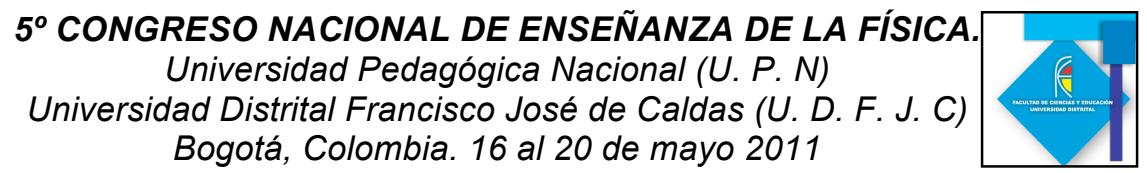

condiciones. Esta forma metodológica que se identifica en Galileo, posibilita al docente la búsqueda de explicaciones de los fenómenos desde los modelos explicativos de los estudiantes, por medio de diálogos, donde la función del docente es orientar sobre las posibles fallas y fortalezas que surgen de dichas explicaciones, construyendo en conjunto

un nuevo modelo explicativo que funcione bajo ciertas condiciones, lo que conlleva a la construcción del conocimiento desde los modos de ver de los estudiantes.

\section{Referencias bibliográficas}

Carrascosa J. (2006) El problema de las concepciones alternativas en la actualidad (parte III). Utilización didáctica de los errores conceptuales que aparecen en cómics, prensa, novelas y libros de texto. Rev. Eureka. Enseñanza y Divulgación de las Ciencias., 3(1), pp. 77-88

Galilei, Galileo (1981 [1638]). Consideraciones y demostraciones matemáticas sobre dos nuevas ciencias. Traducido por J. Sabada. Introducción y notas por C. Solís. Madrid: Editora Nacional, pp 265-379

Valero, Michael (2002). Física Fundamental. Bogotá: Editorial Norma, pp 80

Villegas, Mauricio, Ramírez, Ricardo (1989). Investiguemos Física 10. Bogotá: Editorial Voluntad, pp 80 